\title{
Prediction of oral appliance efficiency in patients with apnoea using phrenic nerve stimulation while awake
}

\author{
Valérie Bosshard, ${ }^{1,2}$ Jean-Franç̧ois Masse, ${ }^{1}$ Frédéric Sériès ${ }^{1}$
}

- Supplementary data is published online. To view this file please visit the journal online (http://thorax.bmj.com).

${ }^{1}$ Centre de recherche de I'Hôpital Laval, Institut Universitaire de cardiologie et de pneumologie de Québec, Québec, Canada

${ }^{2}$ Service de pneumologie, Hôpitaux Universitaires de Genève, Suisse

\section{Correspondence to} Dr Frédéric Sériès, Unité de recherche en pneumologie, Institut universitaire de cardiologie et de pneumologie de Québec, 2725 Chemin Sainte Foy, Sainte Foy (Québec). Canada, G1V 4G5; frederic.series@med.ulaval.ca

Received 31 August 2010 Accepted 16 December 2010 Published Online First 12 January 2011
ABSTRAC

Background In patients with sleep apnoea, primary oropharyngeal collapse of the upper airway during sleep is an important predictor of treatment success with an oral appliance. Phrenic nerve stimulation is able to induce upper airway obstruction and was therefore used to mimic the target for an efficient treatment with an oral appliance.

Objective To test if the site(s) of upper airway collapse identified by means of bilateral anterior magnetic stimulation during wakefulness could predict the efficacy of treatment with an oral appliance in patients with obstructive sleep apnoea.

Methods The site(s) of upper airway collapse while awake were identified by examining the flow-pressure relationship of flow-limited twitches when measuring velopharyngeal and oropharyngeal pressure. Once the mandibular advancement titration had been completed, the efficacy of mandibular advancement was documented during an in-lab sleep study.

Results 33 patients (24 men and 9 women, apnoea-hypopnoea index (AHI) $32.5 \pm 17.1 / \mathrm{h}$ ) participated in the study. Flow limitation was obtained in 29 , but 3 of these had no follow-up sleep study with the device. Subjects with oropharyngeal and velopharyngeal collapse did not differ in the phenotypic characteristics associated with a positive response to an oral appliance (gender, apnoea severity, body mass index or positional dependency of breathing disturbances). Complete or partial success was seen in 14/17 subjects with twitchinduced oropharyngeal collapse and in 4/12 patients with velopharyngeal closure. Treatment response was significantly different in subjects with twitch-induced oropharyngeal and velopharyngeal collapse (OR 9.5, 95\% Cl 1.6 to 52.7).

Conclusions Identifying the site of upper airway collapse by using bilateral anterior magnetic stimulation of the phrenic nerve during wakefulness can predict treatment success with an oral appliance in patients with sleep apnoea.

\section{INTRODUCTION}

Obstructive sleep apnoea (OSA) is highly prevalent in modern societies and is associated with a significant increase in morbidity and mortality due the increase in cardio-metabolic risk and vigilance impairment. Continuous positive airway pressure (CPAP) is the first recommended treatment for OSA since it is the most effective treatment for sleep-induced breathing disturbances. As it acts by pneumatic splinting of the different levels of the upper airway, the effectiveness of CPAP does not depend on the location of upper airway collapse.
However, its clinical effectiveness is limited by its cumbersome nature, and CPAP adherence is always a concern despite continuous improvements in design of the interfaces and in modalities of CPAP machine pressure delivery. For this reason, alternative therapeutic options have been developed and there is growing evidence that mandibular advancement devices (MADs) may represent an effective treatment of OSA. ${ }^{1}$ Indeed, their ability to decrease breathing disturbance indices has been demonstrated, ${ }^{2}$ in terms of improving clinical symptoms (snoring, sleep quality, excessive daytime sleepiness). ${ }^{2} 3$ The simplicity and ambulatory nature of treatment with $\mathrm{MADs}$ may account for the better adherence to MADs than to nasal CPAP. ${ }^{4}$ Unfortunately, the ability of an $M A D$ to normalise breathing at night is not as good as for CPAP. Some anthropometric and polysomnographic parameters have been recognised as good predictors of successful MAD outcome, such as lower age, female gender, lower body mass index (BMI), lower baseline apnoea-hypopnoea index (AHI) and the presence of position dependency of sleep-disordered breathing. ${ }^{5}$ On the other hand, considering that an MAD prevents upper airway closure by moving the mandibule and attached soft tissues forward, the site of the upper airway collapse during sleep is thought to be a powerful predictor of MAD treatment success. ${ }^{67}$ Indeed, the identification of a primary oropharyngeal obstruction during sleep has been found to be associated with a dramatic increase in success rate. $^{8}$

Phrenic nerve stimulation, bilateral anterior magnetic phrenic stimulation (BAMPS), can reproduce during wakefulness the conditions associated with upper airway obstruction during sleep by dissociating the activity of the upper airway and inspiratory muscles. ${ }^{9}$ We have previously found that it may be a useful tool to identify subjects with $\mathrm{OSA}^{10}$ and to determine CPAP settings. ${ }^{11}$ Moreover, this technique provides unique information on upper airway dynamic properties of the nonphasically active upper airway when the stimulus is applied during expiration and can be used to determine the site of obstruction in the upper airway by analysing the pressure-flow relationship at different pharyngeal levels. ${ }^{12} 13$ We hypothesised that the identification of the site of upper airway collapse when awake by means of phrenic nerve stimulation could accurately predict the efficacy of $\mathrm{MAD}$ treatment in patients with OSA.

The aim of our study was to assess the ability of BAMPS to identify subjects who would benefit from treatment with an $\mathrm{MAD}$ to improve or normalise obstructive breathing disturbances while asleep. 


\section{MATERIAL AND METHODS Subjects}

Thirty-three consecutive patients with sleep apnoea in whom mandibular advancement was retained as treatment choice participated in the study. The use of an oral appliance was proposed as a treatment using the same criteria as used in clinical practice: (1) adequate dental condition; (2) absence of any contraindication related to the temporo-mandibular joint condition; (3) mild/moderate sleep apnoea without severe daytime sleepiness that would require immediate effective treatment; or (iv) CPAP failure due to intolerance, side effects or rebutal. Other inclusion criteria included normal nose breathing, stable medical condition, no history of upper airway surgery, absence of anticipated changes in lifestyle habits (weight loss programme and medication) and absence of medication promoting weight gain or altering respiration during sleep. Sleep apnoea status was documented by a polysomnographic recording. The internal review board of our institution approved this protocol, and informed consent was obtained from every subject.

\section{Sleep recording}

Conventional polysomnographic recordings were completed according to published recommendations ${ }^{14}$ (see online repository). Sleep and respiratory variables were scored according to standard criteria. ${ }^{14}$ All electromyography (EMG), flow and pressure tracings were recorded on a microcomputer (Axoscope software 9.0; Axon Instruments, Sunnyvale, CA, USA). The baseline AHI had to be higher than $10 / \mathrm{h}$ for inclusion in the study.

\section{Measurement of upper airway pressures}

Silver cup electrodes were placed on the mid-clavicular line in the seventh to eighth right and left intercostal spaces for surface recording of the right and left costal diaphragmatic EMG activities (Biopac System/Biopac, Santa Barbara, California, USA). Pressure and flow recordings were completed as previously described $^{10-13}$ (see online repository). Pharyngeal pressure was measured with a pressure-tipped catheter (model CT/S X1058, Gaeltec, Hackensack, New Jersey, USA) whose tip was positioned $2 \mathrm{~cm}$ below the soft palate and $4 \mathrm{~cm}$ down in the oropharynx (figure 1). The head position was maintained by an independent adjustable support made of two firm round pillows applied on each side of the occipital region with the head passively lying on these supports. The angle of the supports was adjusted so that the neck was strictly in alignment with the subject's back without anterior/posterior flexion. This allowed a stable and comfortable head/neck support throughout the experiment.

\section{Phrenic nerve stimulation procedure}

BAMPS was performed with two Magstim 200 stimulators (Magstim, Whitland, Dyfed, UK), connected to two $45 \mathrm{~mm}$ figure-of-eight-shaped coils, according to a previously described technique ${ }^{10-1315}$ (see online repository). BAMPS intensity was individually set at a power output sufficient to induce clear flowlimited twitches as well as at a $20 \%$ higher intensity. As upper airway dynamic properties did not differ between the low and $20 \%$ higher stimulation intensities, only the results obtained at the low stimulation intensity will be presented.

Twitch-induced breaths were considered flow limited when instantaneous flow $\left(\dot{V}_{I}\right)$ plateaued or decreased despite a persistent increase in driving pressure $\left(P_{d}\right)$. A $P_{d}$ versus $\dot{V}_{I}$ relationship including a flow value ranging from 0 to $\dot{V}_{\operatorname{Im}}$ in allowed modelling of the upper airway dynamic response of

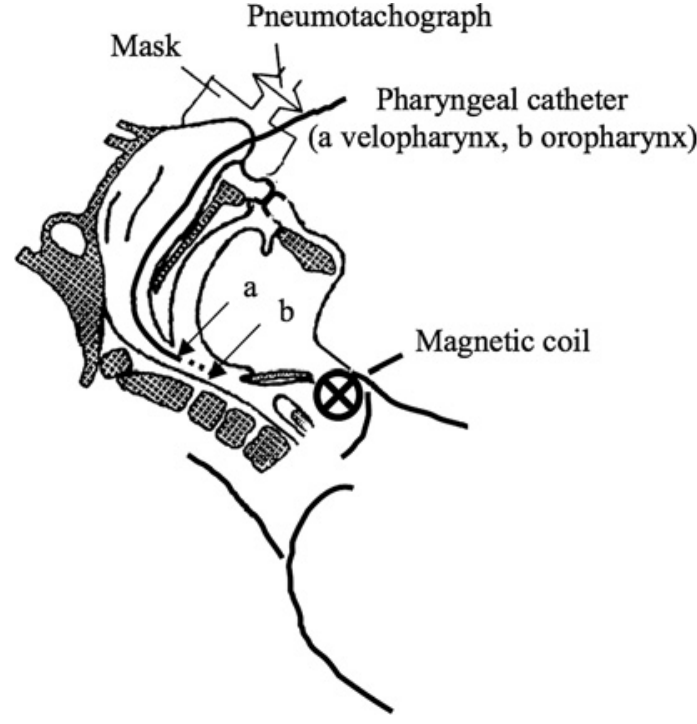

Figure 1 Schematic representation of the experimental set-up. Instantaneous flow was measured with a pneumotachograph connected to the nasal mask. Velopharyngeal and oropharyngeal pressures were recorded with a catheter positioned $2 \mathrm{~cm}$ below the soft palate $(A)$ and $4 \mathrm{~cm}$ (B) down in the oropharynx. Phrenic nerve stimulation was performed with bilateral magnetic anterior stimulation using two figure-of-eight-shaped coils positioned anterolaterally over the anatomical landmark of the phrenic nerve in the neck.

flow-limited twitches with a polynomial regression model of the form $\dot{V}_{I}=k_{1} P_{d}+k_{2} P_{d}^{2}$ as previously described ${ }^{10-13}$ (see online repository). The flow-upper airway pressure relationships fitted well with this polynomial regression model $\left(r^{2} \geq 0.89, p<0.001\right)$. The site(s) of upper airway collapse induced by BAMPS was identified by examining the flow-pressure curves and the $k_{1} / k_{2}$ ratio at the velopharyngeal and oropharyngeal levels. Criteria used to detect the presence of oropharyngeal or velopharyngeal collapse were defined a priori. Exclusive oropharyngeal collapse was identified by the presence of flow limitation exclusively when the catheter was in the distal position (figure 2). In the presence of velopharyngeal collapse, flow limitation was observed at both the upper and lower pressure measurements sites (figure 2). The presence of exclusive velopharyngeal collapse was identified by the occurrence of flow limitation with the catheter below the soft palate and a difference between the $k_{1} / k_{2}$ ratio measured at the oropharyngeal and velopharyngeal levels $\leq 0 \mathrm{~cm} \mathrm{H} \mathrm{H}_{2} \mathrm{O}$. Primary oropharyngeal collapse was identified when this difference was $>1 \mathrm{~cm} \mathrm{H}_{2} \mathrm{O}$. This threshold was chosen according to the reproducibility of twitch-by-twitch closing pressure values obtained in our laboratory and of the variability in velopharyngeal closing pressure values during sleep. ${ }^{8}$

\section{MAD titration procedure}

This was completed by one of the investigators (JFM) after identification of the collapsing site with the phrenic nerve stimulation technique. The choice of the device (ORM, ResMed, SanDiego, California, USA; TAP or Silent Nite, Glidewell Laboratories, Newport Beach, California, USA) was made by this investigator according to his clinical experience. Following an initial acclimatisation period, the $\mathrm{MAD}$ was progressively advanced to obtain an optimal response based on the assessment of subjective (symptoms resolution, abolition of snoring, improvement in daytime sleepiness according to the Epworth 

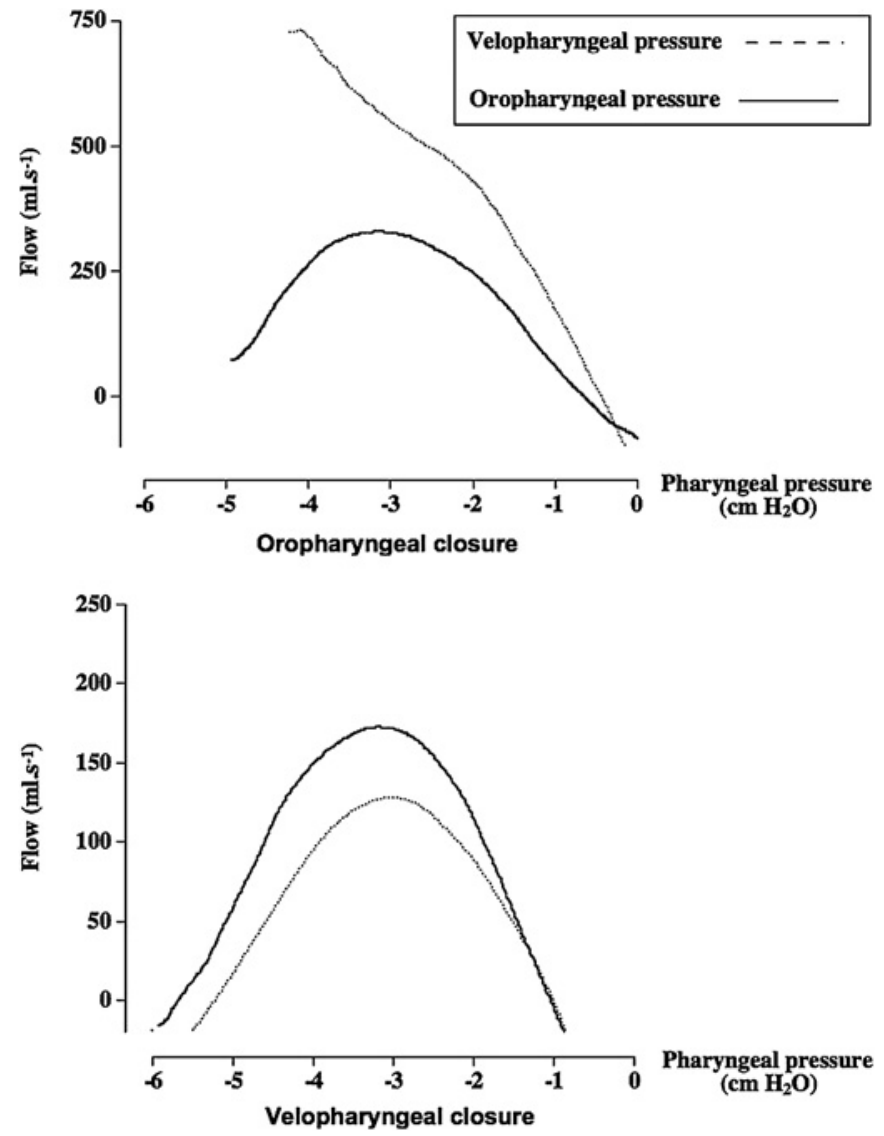

Figure 2 Representative examples of flow-pressure tracings obtained in two subjects demonstrating oropharyngeal and velopharyngeal closure, respectively. Oropharyngeal closure is characterised by the dissociation between twitch flow and oropharyngeal pressure tracings with no plateauing in velopharyngeal pressure. In the case of velopharyngeal closure, the flow-pressure tracings obtained at each site are closely parallel and dissociated from flow tracing.

Sleepiness Score (ESS)) and objective (abbreviated home respiratory recording: Apnoea link, version 6.0, ResMed, Poway, California, USA) ${ }^{16}$ variables or until maximal tolerance.

\section{Study design}

Phrenic nerve stimulation was applied with the catheter positioned at the velopharyngeal or oropharyngeal level and at the two stimulation intensities in random order. Subjects were not instructed regarding the time when the twitch was to be applied. Five twitches were delivered in each condition and two recording sessions were obtained at each upper airway level. The site of upper airway collapse was determined by analysis of the flow-pressure curves (see below). Patients were referred to the dentist for completion of $\mathrm{MAD}$ titration following completion of the phrenic nerve stimulation measurements. After the dentist had determined the optimal amount of mandibular advancement, the efficacy of the MAD was documented by an in-lab sleep study. Subjects were asked to quantify their perceived improvements in snoring, sleep quality and energy on a Likert scale. The dentist and technicians who scored the sleep recording were blind to the results of the BAMPS procedure.

\section{Outcome measures}

Treatment response was evaluated following the control polysomnographic study completed with the MAD in an intention to treat approach. Subjects with an AHI $<10 / \mathrm{h}$ were classified as responders and those with an $\mathrm{AHI} \geq 10$ but with a $>50 \%$ reduction in AHI as partial responders. Treatment failure was defined as the absence of clinical benefit, loss on follow-up and/ or $<50 \%$ reduction in AHI when compared with baseline.

We determined that 21 subjects should be included if $90 \%$ of patients who will improve and $20 \%$ of those who will not improve with an MAD would be identified with phrenic nerve stimulation with a responder/non-responder ratio of $2: 1$ and a $80 \%$ power at a 0.05 significance level. This number was increased to 33 taking into consideration a $25 \%$ procedure failure rate and a $25 \%$ drop-out rate.

\section{Analysis}

Qualitative data are presented as percentages and quantitative data as mean $\pm S D$ or median (min-max). Differences in proportions were tested with the $\chi^{2}$ test or the Fisher exact test, and continuous variables were compared with the Student t test or the Wilcoxon test according to the distribution of the data. The normality assumption was verified with the Shapiro-Wilk test, and the Brown and Forsythe variation of the Levene test statistic was used to verify the homogeneity of variances. Univariate and multivariate logistic regressions were performed to predict the success of the oral appliance in the presence of oropharyngeal closure. Continuous variables were checked for the assumption of linearity in the logit, and the graphical representations suggested linear relationships. The Hosmer-Lemeshow test was performed to assess the goodness-of-fit of the models. The results were considered significant with $p$ values $<0.05$. The data were analysed using the statistical package program SAS v9.2.

\section{RESULTS}

Anthropometric characteristics of participating patients are reported in table 1. An $\mathrm{MAD}$ represented the first-line treatment in 28 subjects while it was indicated due to CPAP intolerance in the other five. The site of upper airway collapse with phrenic nerve stimulation could not be determined in four subjects (intolerance of the procedure in one subject, absence of pressure recording due to technical problems in one and small driving pressure and absence of a flow-limited twitch in the two others). Two other subjects stopped treatment before having completed the $\mathrm{MAD}$ home titration procedure (absence of treatment benefits in one and side effects (excessive salivation) in the other) and one further subject was lost on follow-up. These

Table 1 Characteristics of subjects who completed or did not complete the study

\begin{tabular}{|c|c|c|c|}
\hline & $\begin{array}{l}\text { Study completed } \\
(\mathrm{n}=26)\end{array}$ & $\begin{array}{l}\text { Study not completed } \\
(\mathrm{n}=7)\end{array}$ & p Value \\
\hline Age (years) & $51 \pm 11$ & $52 \pm 10$ & 0.79 \\
\hline Gender (males/females) & $18 / 8$ & $6 / 1$ & 0.64 \\
\hline Baseline BMI $\left(\mathrm{kg} / \mathrm{m}^{2}\right)$ & $27.8 \pm 4.3$ & $27.9 \pm 4.2$ & 0.92 \\
\hline Baseline AHI (n/h) & $30.3 \pm 18.5$ & $29.7 \pm 11.4$ & 0.63 \\
\hline Baseline AHI <30 n/h (\%) & 46.1 & 71.4 & 0.46 \\
\hline SDB position dependency (\%) & 28.0 & 28.6 & 1.0 \\
\hline $\begin{array}{l}\text { Velopharyngeal/oropharyngeal } \\
\text { closure (n) }\end{array}$ & $10 / 16$ & $2 / 1$ & 0.53 \\
\hline Success rate $(\%)$ & 69.2 & 42.9 & 0.19 \\
\hline
\end{tabular}


Table 2 Values of closing pressure measured at the velopharyngeal and oropharyngeal level depending on the main site of twitch-induced upper airway collapse

\begin{tabular}{lll}
\hline & $\boldsymbol{k}_{\mathbf{1}} / \boldsymbol{k}_{\mathbf{2}}$ & \\
\cline { 2 - 3 } & Velopharynx & Oropharynx \\
\hline Exclusive velopharyngeal closure $(\mathrm{n}=12)$ & $-8.5 \pm 2.8$ & \\
Exclusive oropharyngeal closure $(\mathrm{n}=9)$ & & $-7.5 \pm 2.8$ \\
Primary oropharyngeal closure $(\mathrm{n}=8)$ & $-8.9 \pm 2.4$ & $-7.3 \pm 2.6$ \\
\hline
\end{tabular}

three subjects were considered as treatment failures. Therefore, complete results could be obtained in 26 subjects. From these, 11 were treated with an ORM, 11 with a TAP and 4 with a Silent Nite device. Subjects who completed the study can be considered as representative of the whole group since there was no difference in the characteristics of subjects who completed or did not complete the study (table 1). In subjects who did not complete the study, 4 were treated with an ORM and 3 with a TAP. It is noteworthy that all but one subject who had no follow-up study with a MAD had exclusive velopharyngeal closure.

Effective BAMPS intensity ranged from $40 \%$ to $100 \%$ (74 $\pm 19 \%$ max). The site of upper airway collapse was remarkably reproducible between the two recording sessions obtained at the velopharyngeal and oropharyngeal sites (difference in the $k_{1} / k_{2}$ ratio for a given upper airway site $=0.3 \pm 0.7 \mathrm{~cm} \mathrm{H}_{2} \mathrm{O}$ ). Twitch-induced flow limitation occurred exclusively at the velopharyngeal site in 12 subjects, at the oropharyngeal site in 9 others (exclusive oropharyngeal collapse) and in the 8 others the oropharyngeal segment was found to be more collapsible (less negative closing pressure) than the velopharyngeal site (primary oropharyngeal collapse) (table 2).

An MAD was associated with an improvement in AHI, supine AHI, sleep fragmentation, nocturnal desaturation and diurnal somnolence (ESS) with no change in BMI (table 3). Improvement in sleep fragmentation and in subjective assessment of sleep quality and energy were greater in subjects with treatment success than in those with no improvement in breathing disorders (data not shown). Complete or partial success was seen in 14/17 subjects with twitch-induced oropharyngeal collapse and in 4/12 patients with velopharyngeal closure (table 4). Treatment response was significantly different in subjects with twitch-induced oropharyngeal and velopharyngeal collapse (sensitivity 82.4 , 95\% CI $58.9 \%$ to $93.8 \%$; specificity $66.7 \%, 95 \%$ CI $39.1 \%$ to $86.2 \%$; OR $9.5,95 \%$ CI 1.6 to $52.7, \mathrm{p}=0.02)$. This provided a positive likelihood ratio of 2.5 and, given the overall

Table 3 Baseline and control values of sleep and respiratory variables in the 26 patients who completed the study

\begin{tabular}{lllr}
\hline & Baseline & With an MAD & p Value \\
\hline Age (years) & $51 \pm 11$ & & \\
Gender (males/females) & $18 / 8$ & & \\
BMI $\left(\mathrm{kg} / \mathrm{m}^{2}\right)$ & $27.8 \pm 4.3$ & $27.6 \pm 4.4$ & 0.33 \\
AHI (n/h) & $30.3 \pm 16.9$ & $10.9 \pm 8.8$ & $<0.01$ \\
Supine AHI (n/h) & $51.8 \pm 19.1$ & $19.6 \pm 12.6$ & $<0.01$ \\
Time supine (\% TST) & $44.7 \pm 24.4$ & $45.7 \pm 23.8$ & 0.81 \\
ESS & $10.8 \pm 4.9$ & $6.4 \pm 3.3$ & $<0.01$ \\
Sleep efficiency (\%) & $75.3 \pm 15.3$ & $82.8 \pm 9$ & 0.02 \\
\% TST $<90 \%$ Sa0 & & $0.0(0.0-2.1)$ & $<0.01$ \\
Respiratory arousal index $(\mathrm{n} / \mathrm{h})$ & $13.5(6.0-60.8)$ & $5.3(0.4-38.0)$ & $<0.01$ \\
\hline
\end{tabular}

Except for \% TST $<90 \% \mathrm{SaO}_{2}$ and the respiratory arousal index that are expressed as median (min-max), variables are reported as the mean \pm SD.

AHI, apnoea-hypopnoea index; BMI, body mass index; ESS, Epworth Sleepiness Score.
Table 4 Characteristics of patients demonstrating velopharyngeal or oropharyngeal (exclusive or primary) collapsing sites according to BAMPS

\begin{tabular}{llll}
\hline & \multicolumn{2}{l}{ Site of obstruction } & \\
\cline { 2 - 3 } & Velopharynx & Oropharynx & p Value \\
\hline Complete responder $(\mathrm{n}=14)$ & 3 & 11 & 0.03 \\
Partial responder $(\mathrm{n}=4)$ & 1 & 3 & \\
Failure $(\mathrm{n}=11)$ & 8 & 3 & \\
Age (years) & $51 \pm 10$ & $50 \pm 12$ & 0.81 \\
Gender (male/female) & $10 / 2$ & $10 / 7$ & 0.23 \\
Neck circumference $(\mathrm{cm})$ & $38.7 \pm 3.2$ & $37.7 \pm 3.1$ & 0.39 \\
Baseline BMI $\left(\mathrm{kg} / \mathrm{m}^{2}\right)$ & $27.1 \pm 2.4$ & $28.1 \pm 5.1$ & 0.51 \\
Baseline AHI $(\mathrm{n} / \mathrm{h})$ & $33.9 \pm 21.9$ & $27.9 \pm 10.9$ & 0.40 \\
Baseline AHI $<30 \mathrm{n} / \mathrm{h}(\%)$ & 58.3 & 41.2 & 0.46 \\
SDB position dependency $(\%)$ & 33.3 & 23.5 & 0.38 \\
Skeletal class I (n) & 6 & 6 & 0.86 \\
Skeletal class II $(\mathrm{n})$ & 5 & 9 & \\
Skeletal class III $(\mathrm{n})$ & 1 & 2 & \\
\hline
\end{tabular}

AHI, apnoea-hypopnoea index; BAMPS, bilateral anterior magnetic phrenic stimulation; BMI, body mass index; SDB, sleep-disordered breathing.

$63.6 \%$ success rate of MADs, the likelihood of treatment success associated with oropharyngeal collapse increased to $81.4 \%$. On the other hand, given the negative likelihood ratio of 0.26 associated with velopharyngeal closure, the likelihood of treatment failure decreased from $36.4 \%$ to $12.9 \%$. Skeletal class data were available in all but one subject who demonstrated oropharyngeal closure. Skeletal class, neck circumference, pharyngeal grade or mallampati score did not differ significantly between subjects with velopharyngeal or oropharyngeal collapse $(p=0.86)$ (table 4). Interestingly, subjects with oropharyngeal and velopharyngeal collapse did not differ in the phenotypic characteristics that have been reported to be helpful in the identification of good responders to $\mathrm{MAD}$ treatment (gender, apnoea severity, BMI, positional dependency of breathing disturbances) (table 4). Univariate analysis demonstrated no association between these parameters and $\mathrm{MAD}$ response at a $p$ value of 0.2 , indicating that none of these parameters improved the predictive value associated with the identification of the pharyngeal site.

\section{DISCUSSION}

Our results demonstrate that the identification of upper airway collapse during wakefulness using phrenic nerve stimulation is a useful tool to identify patients with sleep apnoea who are good candidates for treatment with an MAD.

Without considering BAMPS results on identification of the collapsing site, the overall success rate of MADs was $69.2 \%$. This appears to be higher than the $52 \%$ average ability of an MAD to decrease the AHI below $10 / \mathrm{h}^{5}$ but is close to that recently reported in other studies. ${ }^{17}$ Such a high success rate can also be accounted for by the use of an abbreviated sleep recording to optimise $\mathrm{MAD}$ titration. ${ }^{16}$ The better success rate previously observed by Fleury et al using this strategy (complete response in $63.6 \%$ and partial response in $18.2 \%$ ) may relate to the difference in the population studied. In our patients, no further adjustment of the MAD could be done in patients with treatment failure. It should be stressed that the proposed phrenic stimulation technique provided relevant measures on upper airway collapse characteristics in the majority of the subjects (30/33), technical failure being observed in only four patients (12\%). This $9 \%$ rate is one-third of the discontinuation rate reported in one of the 
largest studies that evaluated conditions associated with success of oral appliances in OSA. ${ }^{5}$

The criteria used to define treatment success correspond to those currently used the most in the literature. ${ }^{1}$ This choice is clinically relevant when comparing the present results with previously reported treatment success rates. We considered that a definition of treatment success based on an AHI $<5 / \mathrm{h}$ with an $\mathrm{MAD}$ was not relevant with the scoring criteria that were used. These were retained because in these subjects with clinical features of sleep apnoea, breathing abnormalities were not consistently associated with a $4 \% \mathrm{SaO}_{2}$ fall but more often with sleep fragmentation. Considering the improvement in sleep quality and diurnal somnolence that we observed and the beneficial effects of $\mathrm{MAD}$ treatment on arterial pressure and oxidative stress and on endothelial dysfunction reported with AHI values on $\mathrm{MAD}$ at $12 \pm 2 / \mathrm{h}^{18}$ and $19.6 \pm 11.5 / \mathrm{h}$, respectively, ${ }^{19}$ we are confident of the efficacy of the treatment as assessed in our study.

Nasal CPAP and MADs both have their respective advantages and disadvantages. When a guaranty of effectiveness is concerned, CPAP is to be considered as the reference treatment. It also allows patients to have a treatment trial without having to purchase the apparatus. On the other hand, when considering the issue of compliance, this has been found to be higher with a MAD than with CPAP. However, the cost of treatment with a $\mathrm{MAD}$ can influence treatment choice since nocturnal breathing disturbances can persist in $\sim 50 \%$ of patients. ${ }^{1}$ This justifies the need to identify phenotypes prone to predict the response to an $\mathrm{MAD}$, and results from large follow-up studies previously demonstrated that gender, weight, baseline AHI and the supine dependence of breathing disturbances may help to predict treatment response.

Strategies aimed at better identification of responders/nonresponders have also been developed taking into account that advancement of the mandible affects the upper and lower upper airway segments differently. Mandibular advancement induces a larger improvement in oropharyngeal than in velopharyngeal cross-sectional area in both anaesthetised obese and non-obese subjects. ${ }^{20}$ This is in accordance with the dramatic influence of the site of upper airway collapse measured during sleep on $\mathrm{MAD}$ treatment success. Henke et al found that the decrease in AHI was greater in subjects who had upper airway closure below the velopharynx compared with those who had velopharyngeal collapse. ${ }^{6}$ Improvement in oropharyngeal patency with mandibular advancement has also been documented by sleep nasoendoscopy in patients whose AHI improved with an $\mathrm{MAD} .^{21}$ Using a multisensor pressure transducer to identify the site of upper airway collapse during sleep, $\mathrm{Ng}$ et al demonstrated that $100 \%$ of subjects with oropharyngeal obstruction normalised their AHI with a MAD while only $37.5 \%$ of patients with velopharyngeal closure were cured. ${ }^{8}$ Therefore, identifying the site of upper airway collapse during sleep provides unique information on future $\mathrm{MAD}$ treatment response in patients with OSA. However, the need for investigations of upper airway mechanics during sleep reduces the practical applicability of these techniques.

This led to the evaluation of the discriminative nature of tests performed during wakefulness. MRI of the pharynx during tidal breathing and the Müller manoeuvre demonstrate that the pattern of upper airway obstruction without and with an $\mathrm{MAD}$ reflects the response to treatment with an MAD. ${ }^{7}$ In a retrospective analysis, Otsuka et al found that airway space, and oropharyngeal airway cross-sectional area were larger and the position of the mandible relative to the cervical spine was longer in subjects who did not improve with an MAD. ${ }^{22}$ A pilot study performed with functional imaging recently suggested that upper airway modelling may be used to predict outcomes of treatment with MADs, the increase in upper airway volume being correlated with improvement in apnoea severity with MADs. ${ }^{23}$ Finally, the Müller manoeuvre performed during nasopharyngoscopy with mandibular advancement identified that airway collapse was greater in the velopharynx in nonresponders than in responders and was still observed with an MAD. ${ }^{24}$ It should be emphasised that the above-mentioned studies compared upper airway static or dynamic features that would discriminate $\mathrm{MAD}$ responders and non-responders, but the predictive value of the proposed models has not been prospectively validated. In fact, prospective evaluation of procedures such as analysis of features of the inspiratory and expiratory flow-volume curves was not found to be helpful to discriminate between responders and non-responders to MADs. ${ }^{25}$ The effects of an $\mathrm{MAD}$ on the upper airway mechanical properties assessed have been previously reported in normal subjects, demonstrating a decrease in the propensity for the upper airway to collapse with an $\mathrm{MAD} .^{26}$

The results of the recent study of Chan et $a l^{27}$ are interesting to discuss in parallel with the present results. These authors found no difference in MRI-assessed volume of airway or soft tissue structures between responders and non-responders to an $\mathrm{MAD}$. However, a significant enlargement of the upper airway volume was observed with an $\mathrm{MAD}$ in responders mainly due to an increase in the volume and lateral dimension of the velopharynx. Thus the success of an MAD could be influenced by its ability to stabilise pharyngeal structures at the velopharyngeal level. In our study, 8/16 subjects with primary oropharyngeal collapse also demonstrated velopharyngeal closure at lower upper airway pressures. In these subjects, the velopharyngeal closing pressure (as assessed by the $k_{1} / k_{2}$ ratio) did not differ from that measured in patients with primary velopharyngeal closure (table 2). On the other hand, independently of the primary closure level, the velopharyngeal closing pressure was less negative in non-responders $\left(-8.5 \pm 1.8 \mathrm{~cm} \mathrm{H} \mathrm{H}_{2} \mathrm{O}\right)$ than in responders $\left(-9.5 \pm 2.5 \mathrm{~cm} \mathrm{H} \mathrm{H}_{2} \mathrm{O}\right)$. However, this difference did not reach significance. Thus, additional experiments aimed at quantifying the effect of mandibular advancement on velopharyngeal collapsibility and its influence on the response to an $\mathrm{MAD}$ would be worth completing. In this instance, the identification of the upper airway obstruction site with a phrenic nerve stimulation technique while awake inscribes into a clinical investigation strategy where knowledge transfer should result in important applications in sleep apnoea treatment strategies.

We conclude that the identification of the anticipated response to an $\mathrm{MAD}$ by means of phrenic nerve stimulation during wakefulness represents an important clinical step in the management of sleep apnoea. According to the present data, the predictive value of BAMPS-derived identification of the site of upper airway occlusion is higher than that using conventional characterisation of phenotypic patients. Since it does not require patients to buy any device, such a procedure could be very effective in targeting subjects who would or would not benefit from $\mathrm{MAD}$ treatment and could be integrated into the investigation strategy of candidate subjects.

Funding Chaire de Pneumologie JD Bégin de l'Université Laval.

\section{Competing interests None.}

Ethics approval This study was conducted with the approval of the Comité d’Éthique, Institut de Cardiologie et de Pneumologie de Québnec.

Provenance and peer review Not commissioned; externally peer reviewed. 


\section{REFERENCES}

1. Ferguson KA, Cartwright $R$, Rogers $R$, et al. Oral appliances for snoring and obstructive sleep apnea: a review. Sleep 2006;29:244-62.

2. Mehta A, Qian J, Petocz P, et al. A randomized, controlled study of a mandibular advancement splint for obstructive sleep apnea. Am J Respir Crit Care Med 2001;163:1457-61.

3. Barnes M, McEvoy RD, Banks S, et al. Efficacy of positive airway pressure and oral appliance in mild to moderate obstructive sleep apnea. Am J Respir Crit Care Med 2004;170:656-64.

4. Gagnadoux F, Fleury B, Vielle B, et al. Titrated mandibular advancement versus positive airway pressure for sleep apnoea. Eur Respir J 2009;34:914-20.

5. Marklund M, Stenlund H, Franklin KA. Mandibular advancement devices in 630 men and women with obstructive sleep apnea and snoring: tolerability and predictors of treatment success. Chest 2004;125:1270-8.

6. Henke KG, Frantz DE, Kuna ST. An oral elastic mandibular advancement device for obstructive sleep apnea. Am J Respir Crit Care Med 2000;143:942-6.

7. Sanner BM, Heise M, Knoben B, et al. MRI of the pharynx and treatment efficacy of a mandibular advancement device in obstructive sleep apnoea syndrome. Eur Respir $\mathrm{J}$ 2002;20:143-50.

8. $\quad \mathbf{N g}$ AT, Qian J, Cistulli PA. Oropharyngeal collapse predicts treatment response with oral appliance therapy in obstructive sleep apnea. Sleep 2006;29:666-71.

9. Sériès $\mathbf{F}$, Strauss $\mathbf{C}$, Demoule $A$, et al. Assessment of upper airway dynamics in awake sleep apnea patients with phrenic nerve stimulation. Am J Respir Crit Care Med 2000;162:795-800.

10. Verin E, Similowski T, Teixeira A, et al. Discriminative power of phrenic twitchinduced dynamic response for diagnosis of sleep apnea during wakefulness. $J$ Appl Physiol 2003:94:31-7.

11. Vérin $\mathbf{E}$, Similowski T, Series F. Effects of continuous positive airway pressure on upper airway inspiratory dynamics in awake patients with sleep-disordered breathing. J Physiol 2003;546:279-87.

12. Sériès $\mathbf{F}$, Ethier $\mathrm{G}$. Site of phrenic nerve stimulation-induced upper airway collapse: influence of expiratory time. J Appl Physiol 2002;92:665-71.

13. Wang $\mathbf{W}$, Verin $E$, Sériès $F$. Influences of the breathing route on upper airway dynamics properties in normal awake subjects with constant mouth opening. Clin Sci (Lond) 2006;111:349-55

14. Anon. Sleep-related breathing disorders in adults. recommendations for syndrome definition and measurement techniques in clinical research. The Report of an American Academy of Sleep Medicine Task Force. Sleep 1999;22:667-89.
15. Kyroussis D, Hamnegard $\mathrm{CH}$, Polkey Ml, et al. Bilateral magnetic stimluation of the phrenic nerves from an anterolateral approach. Am J Respir Crit Care Med 1996:153:1880-7.

16. Fleury B, Rakotonanahary D, Petelle B, et al. Manidbular advancement titration for obstructive sleep apnea. Chest 2004;125:1761-7.

17. Zeng B, Ng AT, Darendellier MA, et al. Use of flow-volume curves to predict ora appliance treatment outcome in obstructive sleep apnea. Am J Respir Crit Care Med 2007; 175:726-30.

18. Gotsopoulos H, Kelly JJ, Cistulli PA. Oral appliance therapy reduces blood pressure in obstructive sleep apnea: a randomized, controlled trial. Sleep 2004;27 934-41.

19. Itzhaki S, Dorchin H, Clark G, et al. The effects of 1 year treatment with a Herbst mandibular advancement splint on obstructive sleep apnea, oxidative stress, and endothelial function. Chest 2007;131:740-9.

20. Isono S, Tanaka $A$, Tagaito $Y$, et al. Pharyngeal patency in response to advancement of the mandible in obese anesthetized persons. Anesthesiology 1997;87:1055-62.

21. Johal A, Battagel JM, Kotecha BT. Sleep nasendoscopy: a diagnostic tool for predicting treatment success with mandibular advancement splints in obstructive sleep apnoea. Eur J Orthod 2005;27:607-14.

22. Otsuka R, de Almeida FR, Lowe A, et al. A comparison of responders and nonresponders to oral appliance therapy for the treatment of obstructive sleep apnea. Am J Orthod Dentofacial Orthop 2006;129:222-9.

23. De Backer JW, Vanderveken OM, Vos WG, et al. Functional imaging using computational fluid dynamics to predict treatment success of mandibular advancement devices in sleep-disordered breathing. J Biomech 2007;40:3708-14

24. Chan AS, Lee RW, Srinivasan VK, et al. Nasopharyngoscopic evaluation of oral appliance therapy for obstructive sleep apnoea. Eur Respir $J$ 2010;35:836-42.

25. Chan AS, Lee RW, Srinivasan VK, et al. Use of flow-volume curves to predict ora appliance treatment outcome in obstructive sleep apnea: a prospective validation study. Sleep Breath Published Online First: 2010 Jul 29. doi:10.1007/s11325-010-0395-7.

26. Verin $\mathbf{E}$, Petelle $\mathrm{B}$, Raux $\mathrm{M}$, et al. Effects of mandibular advancement on upper airway dynamics in awake normal subjects: a pilot study with phrenic nerve stimulation. Sleep Med 2006;7:368-73.

27. Chan AS, Sutherland K, Schwab RJ, et al. The effect of mandibular advancement on upper airway structure in obstructive sleep apnoea. Thorax 2010;65:726-32

\section{Correction}

D S Stavroulias, K L Lau, I O Oey, et al. Thoracoscopic bullectomy for dyspnoea in emphysema: defining new boundaries. Thorax 2010;65:A137. The authors names appeared incorrectly in this abstract. The names should have appeared as follows: D Stavroulias, K Lau, I Oey, A Nakas, S Rathinam, D A Waller.

Thorax 2011:66:225. doi:10.1136/thx.2010.150987.41corr1 